OPEN ACCESS

Edited by:

Alessandro Stecco,

University of Eastern Piedmont, Italy

Reviewed by:

Massimo Galia,

University of Palermo, Italy

Bilgin Kadri Aribas,

Bülent Ecevit University, Turkey

*Correspondence:

Yungen Gan

gdszgyg-1@126.com

Hongwu Zeng

homerzeng@126.com

${ }^{+}$These authors have contributed equally to this work

Specialty section:

This article was submitted to

Cancer Imaging and

Image-directed Interventions,

a section of the journal

Frontiers in Oncology

Received: 02 December 2021

Accepted: 11 January 2022

Published: 18 February 2022

Citation:

Lin G, Zong X, Li Y, Tan W,

Sun W, Zhang $S$, Gan $Y$ and

Zeng $H$ (2022) Whole-Body

MRI Is an Effective Imaging

Modality for Hematological

Malignancy Treatment Response

Assessment: A Systematic

Review and Meta-Analysis.

Front. Oncol. 12:827777.

doi: 10.3389/fonc.2022.827777

\section{Whole-Body MRI Is an Effective Imaging Modality for Hematological Malignancy Treatment Response Assessment: A Systematic Review and Meta-Analysis}

\author{
Guisen $\mathrm{Lin}^{1 \dagger}$, Xiaodan Zong ${ }^{2 \dagger}$, Yaowen $\mathrm{Li}^{1}$, Weiting $\mathrm{Tan}^{3}$, Weisheng Sun ${ }^{4}$, Siqi Zhang ${ }^{4}$, \\ Yungen Gan ${ }^{1 *}$ and Hongwu Zeng ${ }^{1 *}$

\begin{abstract}
1 Department of Radiology, Shenzhen Children's Hospital, Shenzhen, China, ${ }^{2}$ Department of Radiology, The Third Affiliated Hospital, Sun Yat-sen University (SYSU), Guangzhou, China, ${ }^{3}$ China Medical University, Shenyang, China, 4 Shantou University Medical College, Shantou, China
\end{abstract}

Objectives: To evaluate the diagnostic accuracy of whole-body MRI (WB-MRI) for assessment of hematological malignancies' therapeutic response.

Methods: PubMed, Embase, and Web of Science were searched up to August 2021 to identify studies reporting the diagnostic performance of WB-MRI for the assessment of hematological malignancies' treatment response. A bivariate random-effects model was applied for the generation of the pooled diagnostic performance.

Results: Fourteen studies with 457 patients with lymphoma, multiple myeloma, and sarcoma (very small proportion) were analyzed. Overall pooled sensitivity and specificity of WB-MRI were 0.88 (95\% Cl: 0.73-0.95) and 0.86 (95\% Cl: 0.73-0.93), respectively. Studies using whole-body diffusion-weighted imaging (WB-DWI) showed higher sensitivity than those that did not $(0.94$ vs. $0.55, p=0.02)$. The pooled concordance rate of WB-MRI to assess hematological malignancies' treatment response with reference standard was 0.78 (95\% Cl: 0.59-0.96). WB-MRI and PET/CT showed similar diagnostic performance (sensitivity [0.83 vs. 0.92, $p=0.11]$ and specificity [0.87 vs. $0.76, p=0.73]$ ).

Conclusion: WB-MRI has high diagnostic performance for hematological malignancies' treatment response assessment. The adding of WB-DWI is strongly associated with increased sensitivity.

Keywords: whole-body MRI, diagnostic value, treatment response assessment, hematological malignancies, metaanalysis, lymphoma, multiple myeloma, sarcoma

Abbreviations: CR, complete response; FN, false negative; FP, false positive; PD, progressive disease; PR, partial response; QUADAS-2, Quality Assessment of Diagnostic Accuracy Studies; SD, stable disease; SROCs, summary receiver operating characteristic curves; TN, true negative; TP, true positive; WB-MRI, whole-body magnetic resonance imaging; WB-DWI, whole-body diffusion-weighted imaging. 


\section{INTRODUCTION}

Assessment of cancer treatment effectiveness is the basis for treatment plan adjustment, which provides guidance for clinicians on whether to continue or change treatment strategies. Therapeutic response assessed by imaging is particularly crucial for patients who undergo non-surgical treatment since histological confirmation of the tumor regions is not available. For hematological malignancies that are disseminated, monitoring the treatment response of the whole body is of utmost importance. Currently, this requires not only assessing anatomically changes of tumor size but also monitoring metabolic changes, which could be provided by ${ }^{18} \mathrm{~F}$-FDG PET/ CT before and after treatment (1).

With recent technological advances and the advantage of zero radiation exposure, whole-body MRI (WB-MRI) has been increasingly used as a clinical tool for cancer staging (2) and screening (3). Moreover, WB-MRI has shown great potential for assessing the therapeutic response of cancer (4). Compared with PET/CT that provides metabolic information of tumors, WBMRI can detect restricted proton diffusion when combined with whole-body diffusion-weighted imaging (WB-DWI) (5).

However, controversial results remain for WB-MRI as a surrogate imaging modality for hematological malignancies' treatment response assessment. Some scholars reported that WB-MRI had comparable results for treatment response assessment with PET/CT (6), while others reported an inferior performance compared to PET/CT (7). The correlation between tumor metabolism changes and diffusion was also controversial, with some investigators reporting a positive correlation with PET/CT (8) and others reporting a mismatch result (9). Therefore, the aim of the present study was to perform a systematic review and meta-analysis to evaluate the diagnostic value of WB-MRI for the assessment of hematological malignancies' treatment response.

\section{MATERIALS AND METHODS}

\section{Literature Search}

PubMed, Embase, and Web of Science were searched to identify studies exploring the diagnostic value of WB-MRI for cancer treatment response. The search was focused on the following phases: "whole-body magnetic resonance imaging", "Wholebody MRI", "WB-MRI", "Whole-body diffusion weighted imaging", "Whole-body DWI", "WB-DWI", "treatment response", "therapeutic response", and "response assessment". The search process terminated in August 2021. The detailed search strategy is provided in the Supplementary Material.

\section{Study Selection}

Two reviewers (GL and XZ) independently screened the abstracts of the identified articles. Disagreement was resolved by consulting a third reviewer (YG or HZ). Full text of the articles would be subsequently reviewed if they fulfilled the following criteria: 1) treatment response assessment of any type of hematological malignancies using WB-MRI with or without WB-DWI; and 2) article was written in English. The exclusion criteria of the articles with full text reviewed were as follows: 1 ) study with data insufficient to calculate true positive (TP), true negative (TN), false positive (FP), and false negative (FN) for construction of a $2 \times 2$ contingency table; 2) assessment of treatment response using automatic methods or texture analysis; 3) assessment of metastatic cancers without assessing primary cancer; and 4) treatment response assessment of previously treated cancer. Related citations in eligible articles were assessed for inclusion.

\section{Data Extraction and Quality Assessment}

Two reviewers (WS and SZ) independently extracted data of all included studies with any disagreement resolved through discussion. For all the included studies, authors, year of publication, sample size, mean age of patients, reference standard for treatment response, type of cancers, application of WB-DWI or not, application of contrast enhancement or not, study design (prospective or retrospective), MRI field strength, and data for the diagnostic value of WB-MRI to assess treatment response were extracted. We defined patients with complete response (CR), stringent CR, very good partial response (PR), and $\mathrm{PR}$ as a response to treatment, while progressive disease (PD) and stable disease (SD) were defined as treatment failure. For the construction of the $2 \times 2$ contingency table, TP was defined as the true prediction of response to treatment, FP was defined as the false prediction of response to treatment, TN was defined as the true prediction of treatment failure, and FN was defined as the false prediction of treatment failure. The TP, TN, FP, and FN for PET/CT were also extracted if the study used non-PET/CT-based criteria as the reference standard. For studies that reported the treatment response as $\mathrm{CR}, \mathrm{PR}, \mathrm{SD}$, and $\mathrm{PD}$, the concordance rate of WB-DWI for assessment was calculated, which was defined as the number of true predictions of $\mathrm{CR}, \mathrm{PR}, \mathrm{SD}$, and $\mathrm{PD}$ using WB-MRI, divided by the total number of $C R, P R, S D$, and $P D$ using the reference standard.

Quality Assessment of Diagnostic Accuracy Studies (QUADAS-2) was used to evaluate the quality of the included studies (10). Two reviewers independently evaluated the quality of the studies with any disagreement resolved with consensus.

\section{Statistical Analysis}

All statistical analyses were conducted using STATA version 14.0 (StataCorp, College Station, TX, USA). The pooled sensitivity, specificity of WB-MRI to assess therapeutic response, and the pooled concordance rate of WB-MRI were calculated using a random-effects model (11). The calculation was conducted on a per-patient follow-up basis. The summary receiver operating characteristic curves (SROCs) and the area under the SROCs were generated for evaluating the diagnostic performance. Heterogeneity was assessed by $\mathrm{I}^{2}$. The possible cause of heterogeneity was analyzed using meta-regression. Univariate 
meta-regression was conducted initially. If more than one factor was identified to be associated with the heterogeneity through univariate meta-regression, multivariate meta-regression including all the identified factors was performed subsequently. Multilevel mixed-effects logistic regression was used to compare the diagnostic performance of WB-MRI with PET/CT for assessing treatment response with a significant level of $\mathrm{p}<$ 0.05. Publication bias was assessed using Deek's funnel plot.

\section{RESULTS}

\section{Literature Search and Study Selection}

A total of 929 articles were identified through initial search (Figure 1), of which 469 articles were identified from PubMed, 368 from Embase, 90 from Web of Science, and 2 from the reference list of one identified article. Following duplication removal, 608 articles remained. After reviewing the abstract, 565 articles were excluded. With the remaining 43 articles, 14 studies met the inclusion criteria for the quantitative analysis (12-25).

\section{Basic Characteristics of the Included Studies}

Table 1 summarizes the basic characteristics of the included 14 studies. The present meta-analysis included a total of 457 patients with lymphoma, multiple myeloma, or sarcoma (a very small portion of cases in one study). The total number of follow-up for assessing treatment response was 562. Eight studies analyzed patients with multiple myeloma, 5 analyzed patients with lymphoma, and 1 analyzed patients with lymphoma and sarcoma. Three studies evaluated the diagnostic value of WB-
MRI in children or young adults, while 10 studies focused on adult patients. In one study, the age of patients was not reported, and we assumed most patients to be adult since $86 \%(12 / 14)$ of patients were diagnosed with diffuse large B-cell lymphoma, a type of non-Hodgkin lymphoma commonly diagnosed at age over 60 (26). Five studies obtained WB-MRI with a magnetic field of 3.0 Tesla, and the remaining 9 used 1.5 Tesla. WB-DWI was applied in 10 of the 14 studies. Contrast-enhanced WB-MRI was applied in 5 studies. Eleven of the 14 studies were prospectively designed. The calculation of concordance rate was available in 4 studies. Direct comparison of the diagnostic performance between WB-MRI and PET/CT was available for 6 studies.

\section{Quality Assessment, Heterogeneity, and Publication Bias}

Figure 2 demonstrates the overall quality of the included studies. The majority of the studies had high quality with a low risk of bias. One study had a high risk of bias and high concern of applicability for the index test since the threshold for the apparent diffusion coefficient (ADC) value to differentiate treatment response was not pre-specific. One study had an unclear risk of bias and concern of applicability for the index test since it did not indicate whether the WB-MRI was interpreted without knowledge of the results of the reference standard. The risk of bias for flow and timing was unclear for one study since it did not provide the interval between WB-MRI and the reference standard.

Significant heterogeneity was found for the sensitivity $(\mathrm{Q}=$ 98.83, $\left.\mathrm{I}^{2}=86.85, \mathrm{p}<0.001\right)$ and specificity $\left(\mathrm{Q}=80.81, \mathrm{I}^{2}=83.91\right.$, $\mathrm{p}<0.001)$. However, no threshold effect was identified (proportion of heterogeneity likely due to threshold effect = $0.08)$. Figure 3 is Deek's funnel plot, which shows no publication bias $(\mathrm{p}=0.17)$ among the included studies.

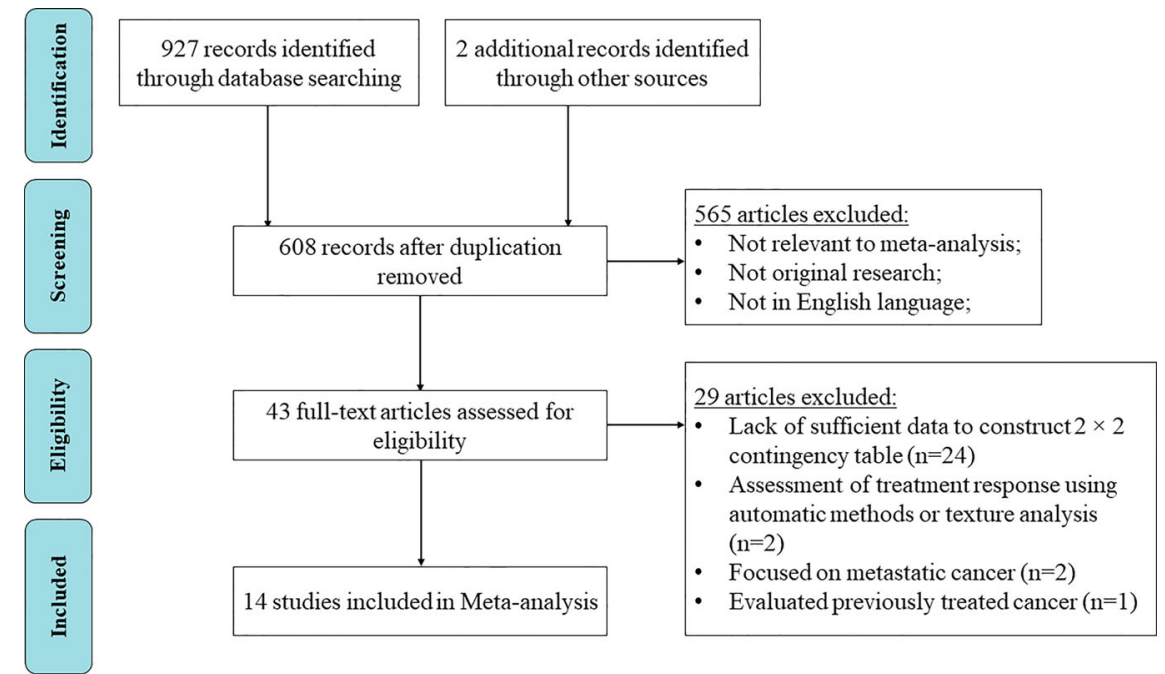

FIGURE 1 | Preferred Reporting Items for Systematic Reviews and Meta-Analyses (PRISMA) flow diagram for article identification and inclusion. 
TABLE 1 | Basic characteristics of the included studies.

\begin{tabular}{|c|c|c|c|c|c|c|c|c|c|}
\hline Author/year & Ref. & $\mathbf{N}$ & Age & Reference standard & Type of cancer & $\begin{array}{l}\text { Magnetic } \\
\text { field (T) }\end{array}$ & $\begin{array}{l}\text { WB- } \\
\text { DWI }\end{array}$ & $\begin{array}{c}\text { Contrast } \\
\text { enhancement }\end{array}$ & $\begin{array}{l}\text { Study } \\
\text { design }\end{array}$ \\
\hline Chieh et al./2010 & $(12)$ & 30 & 58 & IUR criteria for MM & Multiple myeloma & 3 & No & Yes & $P$ \\
\hline Chieh* et al./2010 & (13) & 15 & 48 & Revised IWG criteria & Lymphoma & 3 & Yes & No & $\mathrm{P}$ \\
\hline Marius et al./2011 & (14) & 12 & 61 & IMWG & Multiple myeloma & 3 & Yes & No & $P$ \\
\hline $\begin{array}{l}\text { Suzanne et al./ } \\
2012\end{array}$ & $(15)$ & 51 & 14 & PET/CT-based criteria & Lymphoma & 3 & Yes & No & $\mathrm{P}$ \\
\hline $\begin{array}{l}\text { Thorsten et al./ } \\
2012\end{array}$ & $(16)$ & 31 & 55 & EBMT modified by IUR criteria for MM & Multiple myeloma & 3 & No & Yes & $\mathrm{R}$ \\
\hline $\begin{array}{l}\text { Giuseppe et al./ } \\
2012\end{array}$ & $(17)$ & 29 & $\begin{array}{l}44- \\
83\end{array}$ & IMWG & Multiple myeloma & 3 & No & No & $\mathrm{P}$ \\
\hline Katja et al./2012 & $(18)$ & 14 & NR & Revised IWG criteria & Lymphoma & 1.5 & Yes & No & $\mathrm{P}$ \\
\hline $\begin{array}{l}\text { Sharon et al./ } \\
2014\end{array}$ & (19) & 26 & 61 & IMWG & Multiple myeloma & 3 & Yes & No & $P$ \\
\hline Marius et al./2015 & (20) & 64 & 56 & IHP criteria of the IWG for PET/CT & Lymphoma & 1.5 & Yes & No & $\mathrm{P}$ \\
\hline Arash et al./2018 & $(21)$ & 38 & 16 & PET/CT-based criteria & Lymphoma & 3 & Yes & Yes & $\mathrm{P}$ \\
\hline $\begin{array}{l}\text { Mohammad } \\
\text { et al./2018 }\end{array}$ & (22) & 22 & 62 & IMWG & Multiple myeloma & 1.5 & No & No & $P$ \\
\hline Ho et al./2020 & (23) & 42 & 60 & IMWG & Multiple myeloma & 1.5 & Yes & Yes & $\mathrm{R}$ \\
\hline Ashok et al./2020 & (24) & 56 & 15 & $\begin{array}{l}\text { PET/MRI for lymphoma; PER-CIST and PET/ } \\
\text { MRI for sarcoma }\end{array}$ & $\begin{array}{l}\text { Lymphoma and } \\
\text { Sarcoma }\end{array}$ & 1.5 & Yes & Yes & $P$ \\
\hline $\begin{array}{l}\text { Paternain et al. } \\
\text { /2020 }\end{array}$ & $(25)$ & 27 & 58 & IMWG & Multiple myeloma & 3 & Yes & No & $\mathrm{R}$ \\
\hline
\end{tabular}

EBMT, European Group for Blood and Marrow Transplantation; IHP, International Harmonization Project; IMWG, International Myeloma Working Group; IUR, international uniform response; IWG, International Working Group; N, number (of patients); NR, not reported; P, prospective; PER-CIST, PET response criteria in solid tumors; $R$, retrospective; Ref., reference; T, tesla; WB-MRI, whole-body MRI.

\section{Diagnostic Performance of Whole-Body MRI}

Figure 4 shows the forest plots of the sensitivity and specificity for WB-MRI to assess cancer treatment response. The pooled sensitivity, specificity, positive likelihood ratio, and negative likelihood ratio of WB-MRI for cancer therapeutic response assessment were 0.88 (95\% CI: 0.73-0.95), 0.86 (95\% CI: $0.73-$ 0.93), 6.4 (95\% CI: 3.1-13.4), and 0.14 (95\% CI: 0.06-0.35), respectively. The SROCs are demonstrated in Figure 5 with an area under the curve (AUC) of 0.93 (95\% CI: 0.90-0.95).

\section{Subgroup Analysis and Meta-Regression}

Table 2 represents the result of subgroup analysis and metaregression analysis between each group. In univariate metaregression, the sensitivity of WB-MRI to assess treatment response was significantly higher for studies in patients with lymphoma and sarcoma than in patients with multiple myeloma ( 0.96 vs. $0.76, \mathrm{p}<0.001)$. In addition, the sensitivity was significantly higher for studies using WB-MRI with WB-DWI than those that did not $(0.94$ vs. $0.55, \mathrm{p}=0.02)$. However, when performing multivariate meta-regression, WB-DWI was the only factor related to the heterogeneity $(\mathrm{p}=0.012)$.

\section{Concordance Rate of Whole-Body MRI}

Four studies with a total number of 200 patients and 271 patients' follow-up were available for the concordance rate. Figure 6 shows the forest plot of the concordance rate of WBMRI. The pooled concordance rate of WB-MRI compared with the reference standard was 0.78 (95\% CI: 0.59-0.96).

\section{Whole-Body MRI Versus PET/CT for Cancer Treatment Response Assessment and Contrast-Enhanced Versus No Contrast-Enhanced Whole-Body MRI for Lymphoma Treatment Response Assessment}

Table 3 shows the specific subgroup analysis. Six studies with a total patient number of 179 and 193 patient follow-up were available for the comparison of the diagnostic performance between WB-MRI and PET/CT for cancer treatment assessment. There was no significant difference either for the sensitivity ( 0.83 vs. $0.92, \mathrm{p}=0.11)$ or for the specificity ( 0.87 vs. $0.76, \mathrm{p}=0.73$ ). For treatment assessment of lymphoma, contrastenhanced WB-MRI was applied in 2 studies, while the rest of the 4 studies were conducted without contrast-enhanced sequences. Contrast-enhanced WB-MRI had a higher sensitivity (0.99 vs. $0.79, \mathrm{p}<0.001)$ but a similar specificity $(0.62$ vs. $0.72, \mathrm{p}=0.76)$ for lymphoma treatment assessment as compared to nonenhanced WB-MRI.

\section{DISCUSSION}

The result of the present study showed that WB-MRI had a relatively high sensitivity (0.88) and specificity (0.86) for therapeutic response assessment in patients with lymphoma, multiple myeloma, and sarcoma. The AUC of the SROCs was high (0.93). In addition, we compared the diagnostic performance of WB-MR with PET/CT, which was commonly used as the reference standard for treatment response assessment 


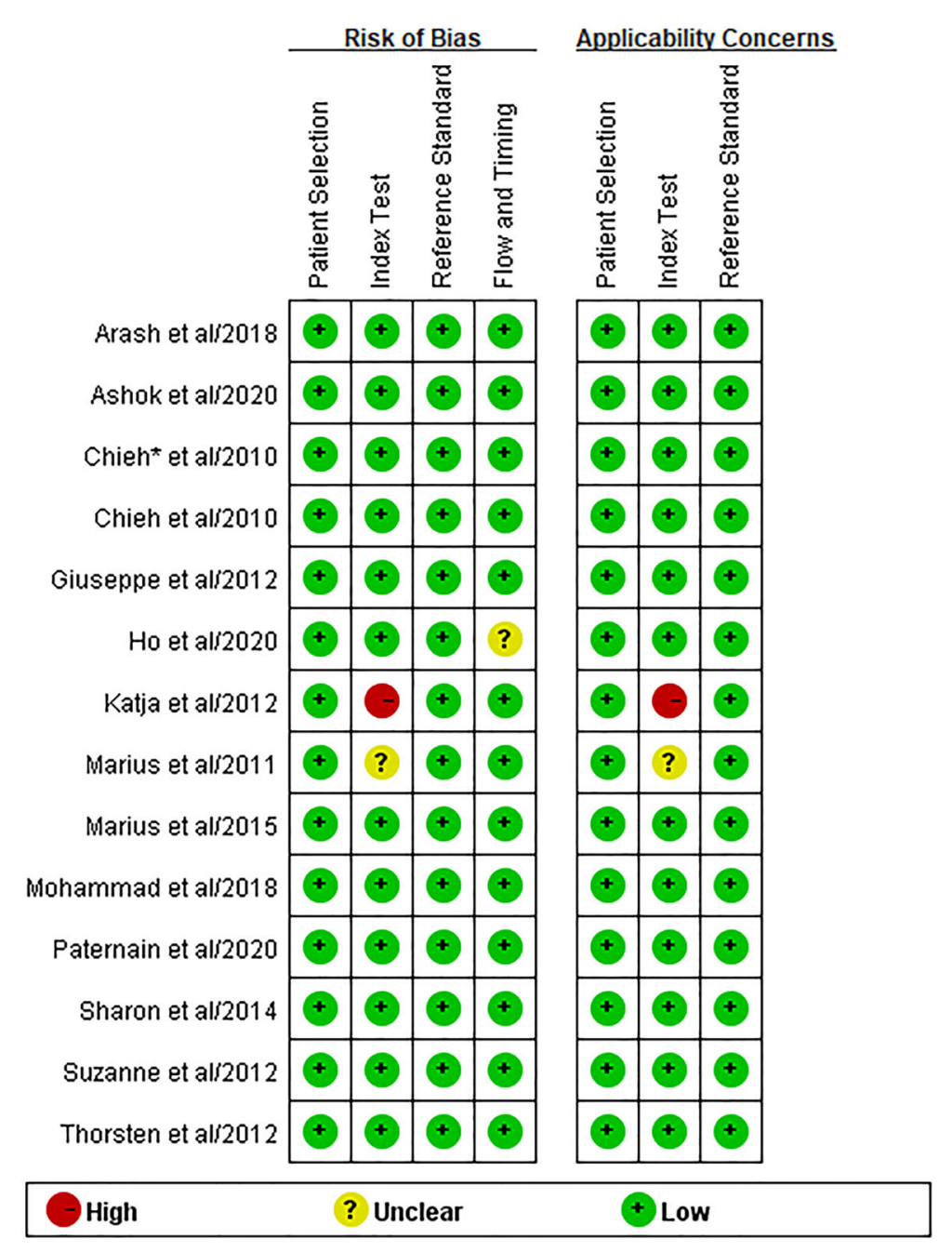

FIGURE 2 | Quality assessment of the included studies using the Quality Assessment of Diagnostic Accuracy Studies (QUADAS-2).

for many types of malignancy $(27,28)$, and we found that there was no significant difference in the sensitivity and specificity. Since the currently used guideline would subclassify the treatment response into CR, PR, SD, and PD (29), we calculated the pooled concordance rate of WB-MRI compared with the reference standard within a subgroup of the studies, which revealed a moderately high concordance rate of $78 \%$. These indicate that WB-MRI is an effective imaging modality for assessing treatment response in patients with lymphoma, multiple myeloma, and sarcoma.

As there was significant heterogeneity for the pooled sensitivity and specificity, subgroup analysis and metaregression were performed. Studies with WB-DWI showed a significantly higher sensitivity for cancer treatment assessment than those without ( 0.94 vs. $0.55, \mathrm{p}=0.02$ at univariate metaregression and $\mathrm{p}=0.012$ at multivariate meta-regression). This is not surprising since DWI is a multipotent imaging modality for cancer diagnosis (30), staging (31), and treatment response assessment (32). Previous studies showed that ADC value increased significantly in responders compared to nonresponders $(5,33)$. Additionally, interim ADC had been shown to help identify non-responder lesions for Hodgkin lymphoma after a few courses of chemotherapy (34). It was believed that the increase of ADC value was due to loss of cellularity caused by effective treatment (35). Therefore, the adding of WB-DWI may increase the sensitivity for the assessment of cancer treatment response. At univariate meta-regression, the sensitivity was significantly higher in patients with lymphoma and sarcoma than patients with multiple myeloma $(0.96$ vs. $0.76, \mathrm{p}<0.001)$. However, at WB-DWI adjusted multivariate meta-regression, no significant difference was found $(\mathrm{p}=0.72)$, which revealed that the difference was caused by whether WB-DWI was applied or not. Indeed, of the 6 studies that focused on multiple myeloma, four of them did not obtain WB-DWI for patients. This addressed the importance of WB-DWI in the assessment of cancer treatment response. 


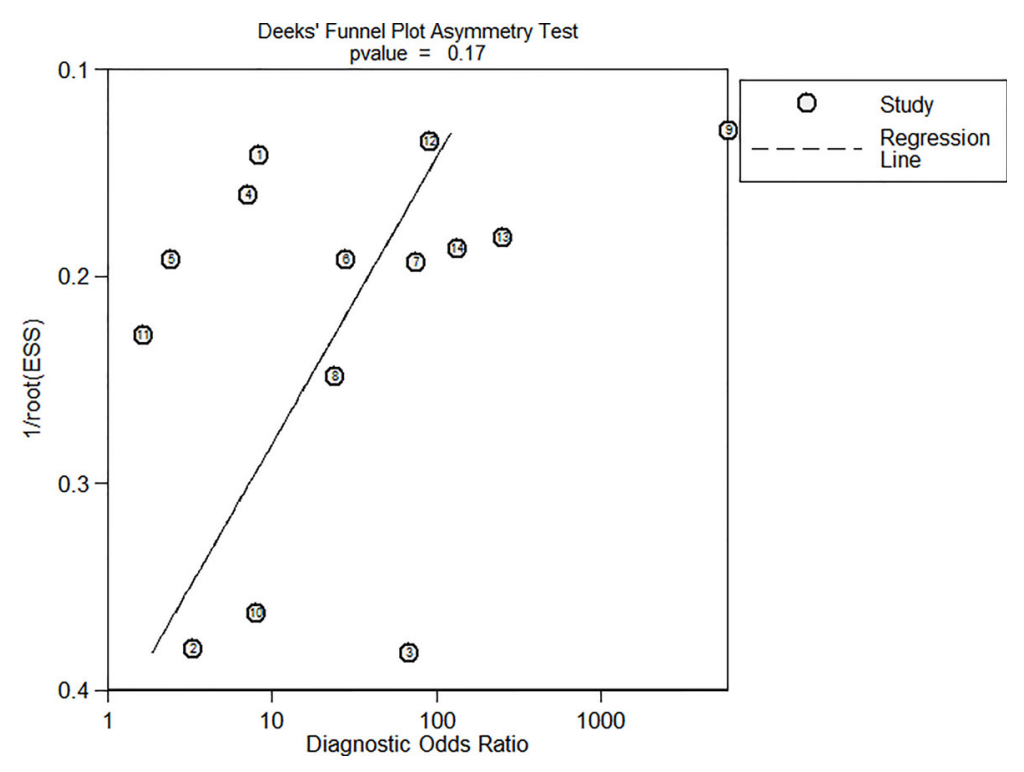

FIGURE 3 | Deek's funnel plot.

The application of contrast-enhanced sequences was not associated with either an increased sensitivity or specificity in the subgroup analysis. However, when we subsequently analyzed WB-MRI for assessment of lymphoma therapeutic response, contrast-enhanced WB-MRI was associated with increased sensitivity $(0.99$ vs. $0.79, \mathrm{p}<0.001)$ but similar specificity $(0.62$ vs. $0.72, \mathrm{p}=0.76)$ as compared to unenhanced WB-MRI. Currently, the use of contrast media for WB-MRI scanning for lymphoma is still being debated (36-38). Previous studies reported that dynamic contrast-enhanced MRI improved the accuracy for detection of splenic involvement of Hodgkin lymphoma (39). The result of the present study might provide evidence for supporting the use of contrast-enhanced sequences in WB-MRI scanning protocols for the assessment of lymphoma treatment response.

In the field of pediatric oncology, WB-MRI is gaining more and more attention. On the one hand, it imparts a zero dose of

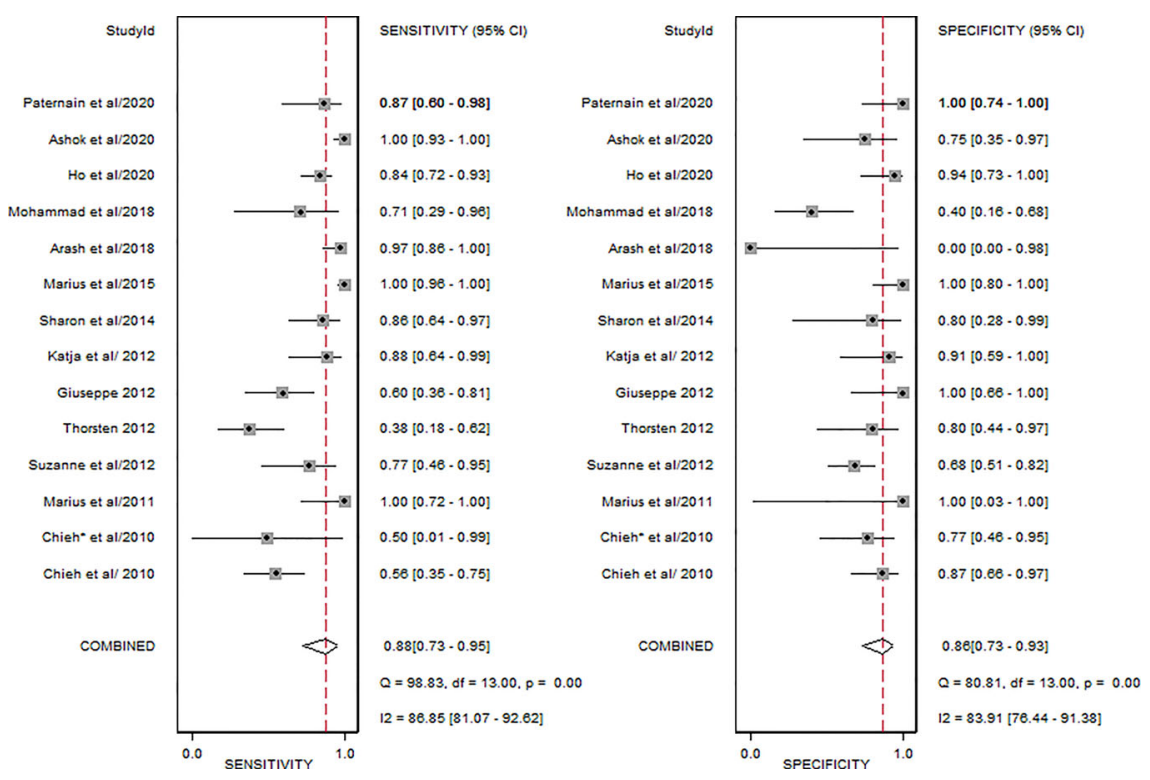

FIGURE 4 | Forest plot of the pooled sensitivity and specificity of the included studies. 


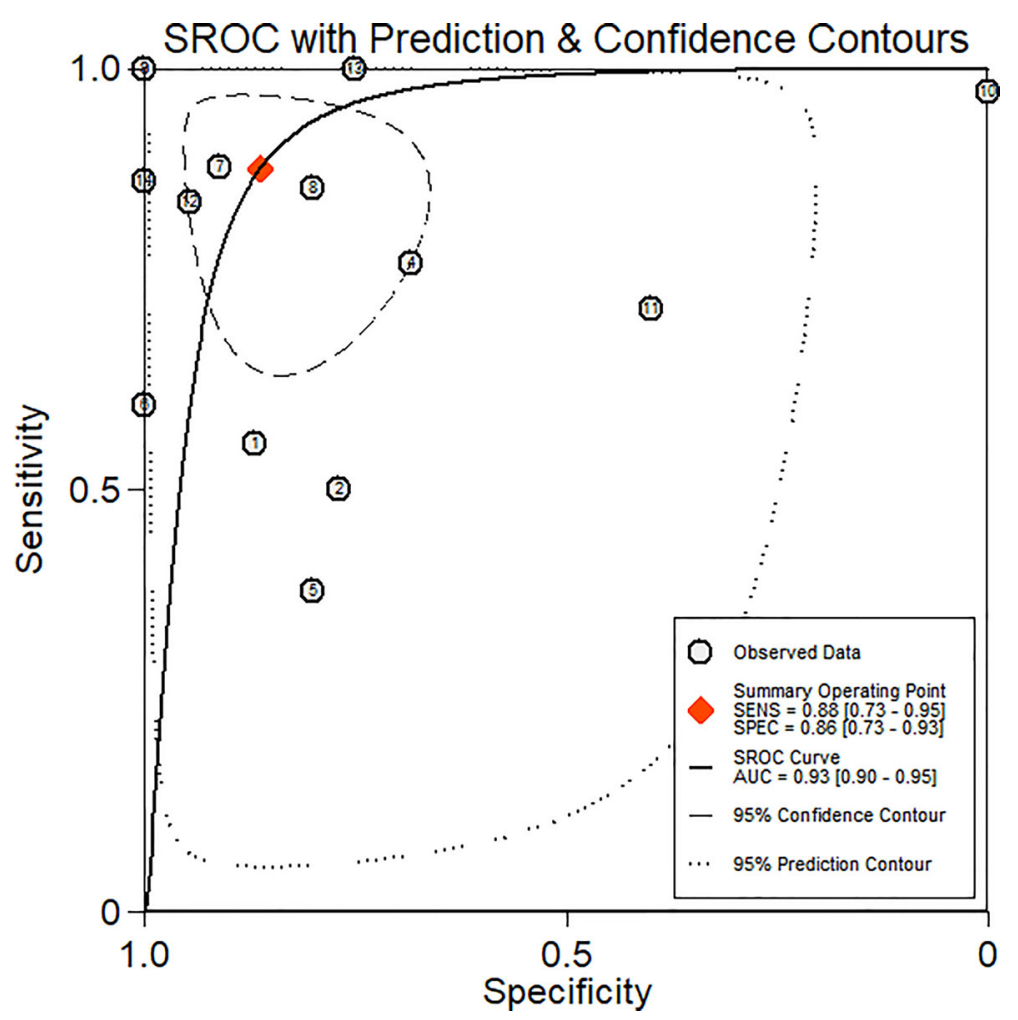

FIGURE 5 | Summary receiver operating characteristics curve.

TABLE 2 | Subgroup analysis and meta-regression.

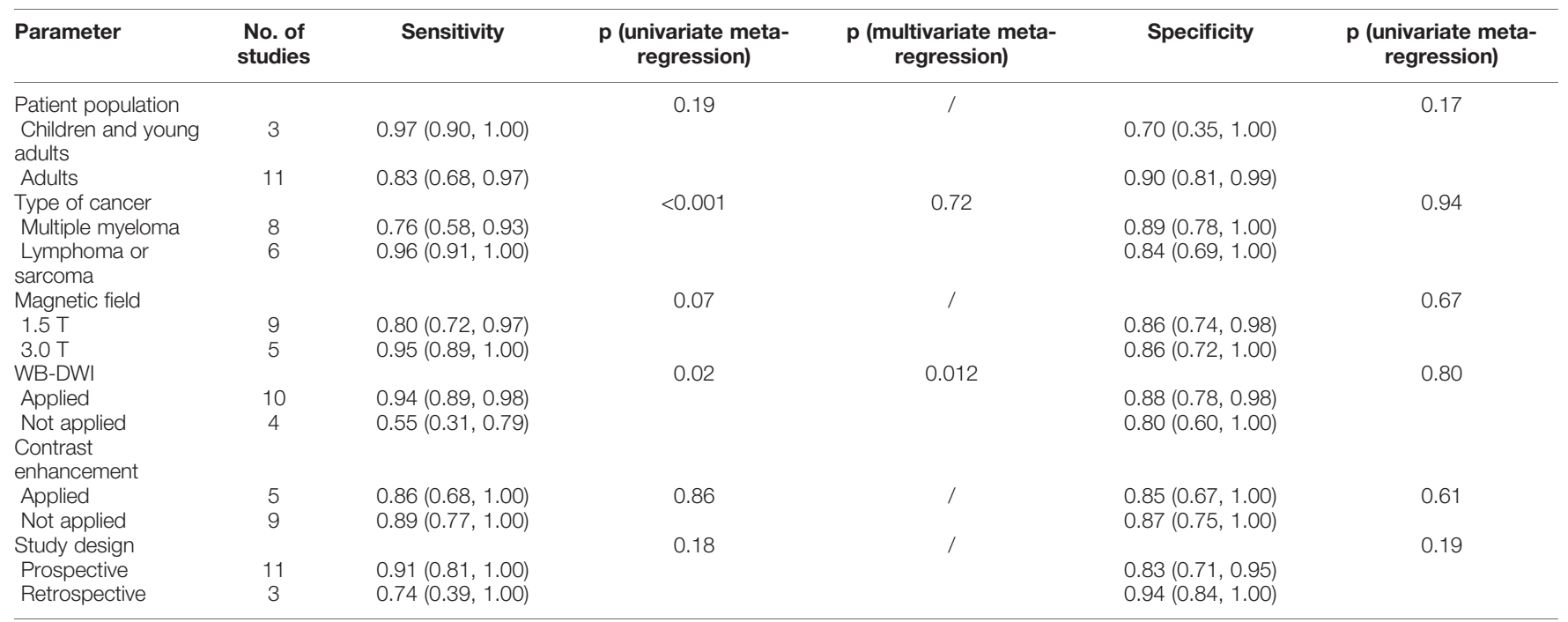

WB-MRI, whole-body MRI.

ionizing radiation, which is particularly important for children and adolescents since their organs and tissues are more radiosensitive (40). On the other hand, WB-MRI could provide high-quality imaging of the entire body within $1 \mathrm{~h}$ (41). The result of the subgroup analysis showed no significant difference in the diagnostic performance for WB-MRI to assess treatment response between the pediatric group and adult group. Of the 3 studies, 2 analyzed pediatric patients with lymphoma, and 1 analyzed pediatric patients with lymphoma (66\%) and sarcoma (34\%). The result of the present study might provide evidence for 
TABLE 3 | Comparison of specific subgroups.

\begin{tabular}{|c|c|c|c|c|c|}
\hline Examination & Number of studies & Sensitivity & $\mathbf{p}$ & Specificity & $\mathbf{p}$ \\
\hline \multicolumn{6}{|l|}{ Whole-body MRI vs. PET/CT } \\
\hline Whole-body MRI & 6 & $0.83(0.54,0.95)$ & 0.11 & $0.87(0.60,0.97)$ & 0.73 \\
\hline $\mathrm{PET} / \mathrm{CT}$ & & $0.92(0.78,0.98)$ & & $0.76(0.62,0.86)$ & \\
\hline \multicolumn{6}{|c|}{ Contrast-enhanced vs. non-enhanced for lymphoma } \\
\hline With contrast enhancement & 6 & $0.99(0.97,1.00)$ & $<0.001$ & $0.62(0.19,1.00)$ & 0.76 \\
\hline Without contrast enhancement & & $0.79(0.96,0.93)$ & & $0.70(0.52,0.87)$ & \\
\hline
\end{tabular}

the use of WB-MRI in pediatric oncology for the treatment assessment of lymphoma and sarcoma.

Previous meta-analyses have shown that WB-MRI had comparable diagnostic performance with PET/CT for distant malignancies (42) and pretherapeutic staging of lymphoma (43). To the best of our knowledge, this is the first metaanalysis to evaluate the diagnostic performance of WB-MRI for hematological malignancies' therapeutic response assessment. Chong et al. have conducted a meta-analysis, which found MRI a potential surrogate imaging modality for treatment response assessment in patients with glioblastoma (44). Moreover, MRI has been suggested for rectal cancer response evaluation to neoadjuvant chemoradiation (45). The present meta-analysis showed that WB-MRI, especially combined with WB-DWI, was a promising imaging tool for treatment response assessment in widely disseminated malignancies such as multiple myeloma, lymphoma, and sarcoma. Plus, it had a comparable diagnostic performance with PET/CT. However, there are still some drawbacks of WB-MRI. First, quantitative assessment of treatment response beyond the mere size evaluation on WBMRI is still challenging. Second, there is no consensus on WBMRI scanning protocols and ADC value thresholds for the assessment of treatment response (36). Third, WB-MRI has some challenging locations in which a miscalculation of ADC measurements may hamper the correct evaluation of ADC values of lymph nodes, particularly in the mediastinum, which is a very common location of lymphoma (46). In comparison, interim PET/CT has a consolidated prognostic role in hematological malignancies, especially in Hodgkin lymphoma where a PETdriven therapy is routinely accepted and performed using FDG uptake to understand the tumor's chemosensitivity (37).

There were several limitations of our meta-analysis. First, the included studies had significant heterogeneity while generating the diagnostic parameters, which might decrease the general applicability of the pooled estimates. However, through univariate and multivariate meta-regression, we identified the cause of the heterogeneity and addressed the importance of applying WB-DWI in WB-MRI for cancer treatment response assessment. Second, the composition of the patient population was heterogeneous, with most patients with hematogenic tumors and a small number of patients with sarcoma. But in subgroup analysis and meta-regression, no significant difference was found for the diagnostic performance of WB-MRI for different types of tumors. Third, we only included studies written in English, which might lead to Tower of Babel bias (47).

In conclusion, WB-MRI has good sensitivity and specificity for the evaluation of the treatment response of multiple myeloma, lymphoma, and sarcoma. The adding of WB-DWI

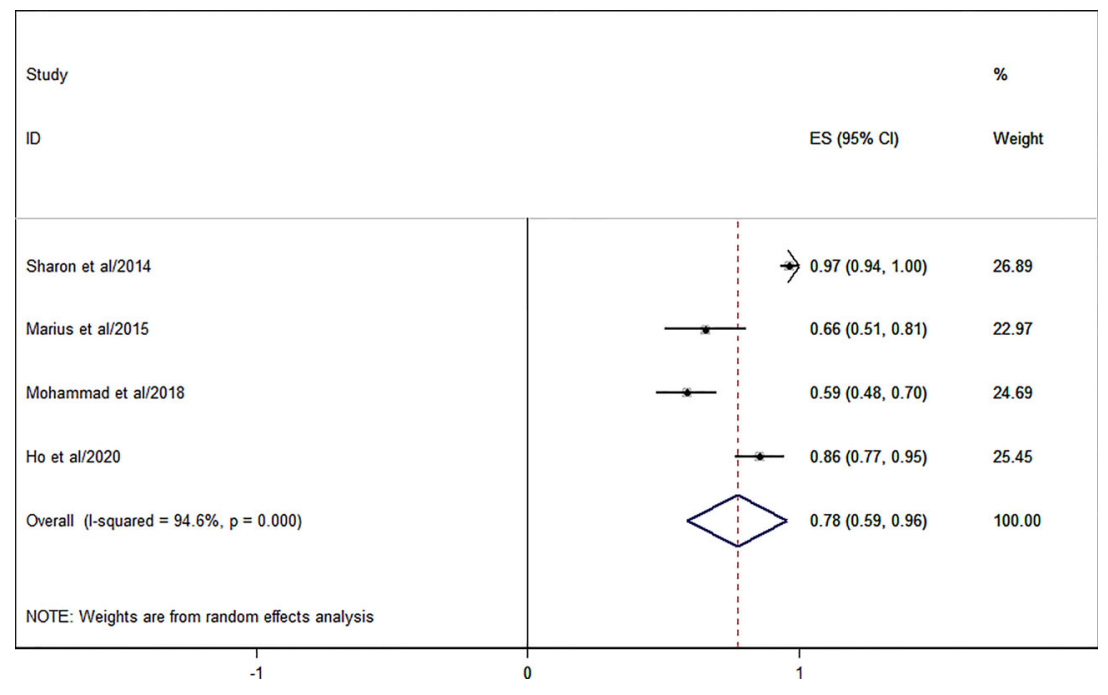

FIGURE 6 | Forest plot of the pooled concordance rate for whole-body MRI (WB-MRI) compared to reference standard. 
would further increase the sensitivity. Additionally, WB-MRI may have comparable performance with PET/CT for therapeutic response assessment, which is particularly attractive in the field of pediatric oncology.

\section{DATA AVAILABILITY STATEMENT}

The original contributions presented in the study are included in the article/Supplementary Material. Further inquiries can be directed to the corresponding authors.

\section{AUTHOR CONTRIBUTIONS}

GL, YG, and HZ designed the study. GL, XZ, YL, and WT performed the literature search. GL and XZ reviewed the articles.

\section{REFERENCES}

1. Wahl RL, Jacene H, Kasamon Y, Lodge MA. From RECIST to PERCIST: Evolving Considerations for PET Response Criteria in Solid Tumors. J Nucl Med (2009) 50 Suppl 1:122S-50S. doi: 10.2967/jnumed.108.057307

2. Taylor SA, Mallett S, Beare S, Bhatnagar G, Blunt D, Boavida P, et al. Diagnostic Accuracy of Whole-Body MRI Versus Standard Imaging Pathways for Metastatic Disease in Newly Diagnosed Colorectal Cancer: The Prospective Streamline C Trial. Lancet Gastroenterol Hepatol (2019) 4:52937. doi: 10.1016/S2468-1253(19)30056-1

3. Zugni F, Padhani AR, Koh DM, Summers PE, Bellomi M, Petralia G. Whole-Body Magnetic Resonance Imaging (WB-MRI) for Cancer Screening in Asymptomatic Subjects of the General Population: Review and Recommendations. Cancer Imaging (2020) 20:34. doi: 10.1186/s40644-020-00315-0

4. Tunariu N, Blackledge M, Messiou C, Petralia G, Padhani A, Curcean S, et al. What's New for Clinical Whole-Body MRI (WB-MRI) in the 21st Century. $\mathrm{Br}$ J Radiol (2020) 93:20200562. doi: 10.1259/bjr.20200562

5. Bonaffini PA, Ippolito D, Casiraghi A, Besostri V, Franzesi CT, Sironi S. Apparent Diffusion Coefficient Maps Integrated in Whole-Body MRI Examination for the Evaluation of Tumor Response to Chemotherapy in Patients With Multiple Myeloma. Acad Radiol (2015) 22:1163-71. doi: 10.1016/j.acra.2015.05.011

6. Siegel MJ, Jokerst CE, Rajderkar D, Hildebolt CF, Goyal S, Dehdashti F, et al. Diffusion-Weighted MRI for Staging and Evaluation of Response in Diffuse Large B-Cell Lymphoma: A Pilot Study. NMR BioMed (2014) 27:681-91. doi: $10.1002 / \mathrm{nbm} .3105$

7. Hagtvedt T, Seierstad T, Lund KV, Londalen AM, Bogsrud TV, Smith HJ, et al. Diffusion-Weighted MRI Compared to FDG PET/CT for Assessment of Early Treatment Response in Lymphoma. Acta Radiol (2015) 56:152-8. doi: $10.1177 / 0284185114526087$

8. Wu X, Kellokumpu-Lehtinen PL, Pertovaara H, Korkola P, Soimakallio S, Eskola H, et al. Diffusion-Weighted MRI in Early Chemotherapy Response Evaluation of Patients With Diffuse Large B-Cell Lymphoma-A Pilot Study: Comparison With 2-Deoxy-2-Fluoro- D-Glucose-Positron Emission Tomography/Computed Tomography. NMR BioMed (2011) 24:1181-90. doi: $10.1002 / \mathrm{nbm} .1689$

9. Rasmussen JH, Norgaard M, Hansen AE, Vogelius IR, Aznar MC, Johannesen $\mathrm{HH}$, et al. Feasibility of Multiparametric Imaging With PET/MR in Head and Neck Squamous Cell Carcinoma. J Nucl Med (2017) 58:69-74. doi: 10.2967/ jnumed.116.180091

10. Whiting PF, Rutjes AW, Westwood ME, Mallett S, Deeks JJ, Reitsma JB, et al. QUADAS-2: A Revised Tool for the Quality Assessment of Diagnostic Accuracy Studies. Ann Intern Med (2011) 155:529-36. doi: 10.7326/00034819-155-8-201110180-00009

11. Reitsma JB, Glas AS, Rutjes AW, Scholten RJ, Bossuyt PM, Zwinderman AH. Bivariate Analysis of Sensitivity and Specificity Produces Informative
WS and SZ acquired the data. YL, WT, WS, and SZ performed the statistical analysis. GL, YG, and HZ drafted the manuscript. All authors revised and approved the final version of the manuscript.

\section{FUNDING}

This study has received funding from the Sanming Project of Medicine in Shenzhen (No. SZSM202011005).

\section{SUPPLEMENTARY MATERIAL}

The Supplementary Material for this article can be found online at: https://www.frontiersin.org/articles/10.3389/fonc.2022.827777/ full\#supplementary-material

Summary Measures in Diagnostic Reviews. J Clin Epidemiol (2005) 58:98290. doi: 10.1016/j.jclinepi.2005.02.022

12. Lin C, Luciani A, Belhadj K, Deux JF, Kuhnowski F, Maatouk M, et al Multiple Myeloma Treatment Response Assessment With Whole-Body Dynamic Contrast-Enhanced MR Imaging. Radiology (2010) 254:521-31. doi: 10.1148/radiol.09090629

13. Lin C, Itti E, Luciani A, Zegai B, Lin SJ, Kuhnowski F, et al. Whole-Body Diffusion-Weighted Imaging With Apparent Diffusion Coefficient Mapping for Treatment Response Assessment in Patients With Diffuse Large B-Cell Lymphoma: Pilot Study. Invest Radiol (2011) 46:341-9. doi: 10.1097/ RLI.0b013e3182087b03

14. Horger M, Weisel K, Horger W, Mroue A, Fenchel M, Lichy M. Whole-Body Diffusion-Weighted MRI With Apparent Diffusion Coefficient Mapping for Early Response Monitoring in Multiple Myeloma: Preliminary Results. AJR Am J Roentgenol (2011) 196:W790-5. doi: 10.2214/AJR.10.5979

15. Spijkers S, Littooij AS, Kwee TC, Tolboom N, Beishuizen A, Bruin MCA, et al. Whole-Body MRI Versus an [(18)F]FDG-PET/CT-Based Reference Standard for Early Response Assessment and Restaging of Paediatric Hodgkin's Lymphoma: A Prospective Multicentre Study. Eur Radiol (2021) 31:892536. doi: 10.1007/s00330-021-08026-1

16. Derlin T, Peldschus K, Munster S, Bannas P, Herrmann J, Stubig T, et al. Comparative Diagnostic Performance of (1)(8)F-FDG PET/CT Versus Whole-Body MRI for Determination of Remission Status in Multiple Myeloma After Stem Cell Transplantation. Eur Radiol (2013) 23:570-8. doi: 10.1007/s00330-012-2600-5

17. Cascini GL, Falcone C, Console D, Restuccia A, Rossi M, Parlati A, et al. Whole-body MRI And PET/CT in Multiple Myeloma Patients During Staging and After Treatment: Personal Experience in a Longitudinal Study. Radiol Med (2013) 118:930-48. doi: 10.1007/s11547-013-0946-7

18. De Paepe K, Bevernage C, De Keyzer F, Wolter P, Gheysens O, Janssens A, et al. Whole-Body Diffusion-Weighted Magnetic Resonance Imaging at 3 Tesla for Early Assessment of Treatment Response in Non-Hodgkin Lymphoma: A Pilot Study. Cancer Imaging (2013) 13:53-62. doi: 10.1102/ 1470-7330.2013.0006

19. Giles SL, Messiou C, Collins DJ, Morgan VA, Simpkin CJ, West S, et al. Whole-Body Diffusion-Weighted MR Imaging for Assessment of Treatment Response in Myeloma. Radiology (2014) 271:785-94. doi: 10.1148/ radiol.13131529

20. Mayerhoefer ME, Karanikas G, Kletter K, Prosch H, Kiesewetter B, Skrabs C, et al. Evaluation of Diffusion-Weighted Magnetic Resonance Imaging for Follow-Up and Treatment Response Assessment of Lymphoma: Results of an 18F-FDG-PET/CT-Controlled Prospective Study in 64 Patients. Clin Cancer Res (2015) 21:2506-13. doi: 10.1158/1078-0432.CCR-14-2454

21. Latifoltojar A, Punwani S, Lopes A, Humphries PD, Klusmann M, Menezes LJ, et al. Whole-Body MRI for Staging and Interim Response Monitoring in Paediatric and Adolescent Hodgkin's Lymphoma: A Comparison With Multi- 
Modality Reference Standard Including (18)F-FDG-PET-Ct. Eur Radiol (2019) 29:202-12. doi: 10.1007/s00330-018-5445-8

22. Basha MAA, Hamed MAG, Refaat R, AlAzzazy MZ, Bessar MA, Mohamed EM, et al. Diagnostic Performance of (18)F-FDG PET/CT and Whole-Body MRI Before and Early After Treatment of Multiple Myeloma: A Prospective Comparative Study. Jpn J Radiol (2018) 36:382-93. doi: 10.1007/s11604-0180738-z

23. Park HY, Kim KW, Yoon MA, Lee MH, Chae EJ, Lee JH, et al. Role of WholeBody MRI for Treatment Response Assessment in Multiple Myeloma: Comparison Between Clinical Response and Imaging Response. Cancer Imaging (2020) 20:14. doi: 10.1186/s40644-020-0293-6

24. Theruvath AJ, Siedek F, Muehe AM, Garcia-Diaz J, Kirchner J, Martin O, et al. Therapy Response Assessment of Pediatric Tumors With Whole-Body Diffusion-Weighted MRI and FDG PET/MRI. Radiology (2020) 296:143-51. doi: 10.1148/radiol.2020192508

25. Paternain A, Garcia-Velloso MJ, Rosales JJ, Ezponda A, Soriano I, Elorz M, et al. The Utility of ADC Value in Diffusion-Weighted Whole-Body MRI in the Follow-Up of Patients With Multiple Myeloma. Correlation Study With (18)F-FDG PET-CT. Eur J Radiol (2020) 133:109403. doi: 10.1016/ j.ejrad.2020.109403

26. Di M, Huntington SF, Olszewski AJ. Challenges and Opportunities in the Management of Diffuse Large B-Cell Lymphoma in Older Patients. Oncologist (2021) 26:120-32. doi: 10.1002/onco.13610

27. Zaucha JM, Chauvie S, Zaucha R, Biggii A, Gallamini A. The Role of PET/CT in the Modern Treatment of Hodgkin Lymphoma. Cancer Treat Rev (2019) 77:44-56. doi: 10.1016/j.ctrv.2019.06.002

28. Cavo M, Terpos E, Nanni C, Moreau P, Lentzsch S, Zweegman S, et al. Role of (18)F-FDG PET/CT in the Diagnosis and Management of Multiple Myeloma and Other Plasma Cell Disorders: A Consensus Statement by the International Myeloma Working Group. Lancet Oncol (2017) 18:e206-17. doi: 10.1016/ S1470-2045(17)30189-4

29. Therasse P, Arbuck SG, Eisenhauer EA, Wanders J, Kaplan RS, Rubinstein L, et al. New Guidelines to Evaluate the Response to Treatment in Solid Tumors. European Organization for Research and Treatment of Cancer, National Cancer Institute of the United States, National Cancer Institute of Canada. J Natl Cancer Inst (2000) 92:205-16. doi: 10.1093/jnci/92.3.205

30. Lan H, Lin G, Zhong W. A Meta-Analysis of the Added Value of Diffusion Weighted Imaging in Combination With Contrast-Enhanced Magnetic Resonance Imaging for the Diagnosis of Small Hepatocellular Carcinoma Lesser or Equal to $2 \mathrm{Cm}$. Oncol Lett (2020) 20:2739-48. doi: 10.3892/ol.2020.11805

31. Regacini R, Puchnick A, Shigueoka DC, Iared W, Lederman HM. Whole-Body Diffusion-Weighted Magnetic Resonance Imaging Versus FDG-PET/CT for Initial Lymphoma Staging: Systematic Review on Diagnostic Test Accuracy Studies. Sao Paulo Med J (2015) 133:141-50. doi: 10.1590/1516-3180.2014.8312810

32. van der Hoorn A, van Laar PJ, Holtman GA, Westerlaan HE. Diagnostic Accuracy of Magnetic Resonance Imaging Techniques for Treatment Response Evaluation in Patients With Head and Neck Tumors, a Systematic Review and Meta-Analysis. PloS One (2017) 12:e0177986. doi: 10.1371/journal.pone.0177986

33. Horger M, Claussen C, Kramer U, Fenchel M, Lichy M, Kaufmann S. Very Early Indicators of Response to Systemic Therapy in Lymphoma Patients Based on Alterations in Water Diffusivity-A Preliminary Experience in 20 Patients Undergoing Whole-Body Diffusion-Weighted Imaging. Eur J Radiol (2014) 83:1655-64. doi: 10.1016/j.ejrad.2014.05.027

34. Albano D, Patti C, Matranga D, Lagalla R, Midiri M, Galia M. Whole-Body Diffusion-Weighted MR and FDG-PET/CT in Hodgkin Lymphoma: Predictive Role Before Treatment and Early Assessment After Two Courses of ABVD. Eur J Radiol (2018) 103:90-8. doi: 10.1016/j.ejrad.2018.04.014

35. Kwee TC, Takahara T, Ochiai R, Katahira K, Van Cauteren M, Imai Y, et al. Whole-Body Diffusion-Weighted Magnetic Resonance Imaging. Eur J Radiol (2009) 70:409-17. doi: 10.1016/j.ejrad.2009.03.054
36. Albano D, Stecco A, Micci G, Sconfienza LM, Colagrande S, Reginelli A, et al. Whole-Body Magnetic Resonance Imaging (WB-MRI) in Oncology: An Italian Survey. Radiol Med (2021) 126:299-305. doi: 10.1007/s11547-02001242-7

37. Albano D, Micci G, Patti C, Midiri F, Albano S, Lo Re G, et al. Whole-Body Magnetic Resonance Imaging: Current Role in Patients With Lymphoma. Diagn (Basel) (2021) 11:1007. doi: 10.3390/diagnostics11061007

38. Albano D, Bruno A, Patti C, Micci G, Midiri M, Tarella C, et al. Whole-Body Magnetic Resonance Imaging (WB-MRI) in Lymphoma: State of the Art. Hematol Oncol (2020) 38:12-21. doi: 10.1002/hon.2676

39. Punwani S, Cheung KK, Skipper N, Bell N, Bainbridge A, Taylor SA, et al. Dynamic Contrast-Enhanced MRI Improves Accuracy for Detecting Focal Splenic Involvement in Children and Adolescents With Hodgkin Disease. Pediatr Radiol (2013) 43:941-9. doi: 10.1007/s00247-012-2616-7

40. Nievelstein RA, Littooij AS. Whole-Body MRI in Paediatric Oncology. Radiol Med (2016) 121:442-53. doi: 10.1007/s11547-015-0600-7

41. Davis JT, Kwatra N, Schooler GR. Pediatric Whole-Body MRI: A Review of Current Imaging Techniques and Clinical Applications. J Magn Reson Imaging (2016) 44:783-93. doi: 10.1002/jmri.25259

42. Xu GZ, Li CY, Zhao L, He ZY. Comparison of FDG Whole-Body PET/CT and Gadolinium-Enhanced Whole-Body MRI for Distant Malignancies in Patients With Malignant Tumors: A Meta-Analysis. Ann Oncol (2013) 24:96-101. doi: 10.1093/annonc/mds234

43. Wang D, Huo Y, Chen S, Wang H, Ding Y, Zhu X, et al. Whole-Body MRI Versus (18)F-FDG PET/CT for Pretherapeutic Assessment and Staging of Lymphoma: A Meta-Analysis. Onco Targets Ther (2018) 11:3597-608. doi: 10.2147/OTT.S148189

44. Suh CH, Kim HS, Jung SC, Choi CG, Kim SJ. Multiparametric MRI as a Potential Surrogate Endpoint for Decision-Making in Early Treatment Response Following Concurrent Chemoradiotherapy in Patients With Newly Diagnosed Glioblastoma: A Systematic Review and Meta-Analysis. Eur Radiol (2018) 28:2628-38. doi: 10.1007/s00330-017-5262-5

45. Kalisz KR, Enzerra MD, Paspulati RM. MRI Evaluation of the Response of Rectal Cancer to Neoadjuvant Chemoradiation Therapy. Radiographics (2019) 39:538-56. doi: 10.1148/rg.2019180075

46. Albano D, La Grutta L, Grassedonio E, Patti C, Lagalla R, Midiri M, et al. Pitfalls in Whole Body MRI With Diffusion Weighted Imaging Performed on Patients With Lymphoma: What Radiologists Should Know. Magn Reson Imaging (2016) 34:922-31. doi: 10.1016/j.mri.2016.04.023

47. Gregoire G, Derderian F, Le Lorier J. Selecting the Language of the Publications Included in a Meta-Analysis: Is There a Tower of Babel Bias? J Clin Epidemiol (1995) 48:159-63. doi: 10.1016/0895-4356(94) 00098-B

Conflict of Interest: The authors declare that the research was conducted in the absence of any commercial or financial relationships that could be construed as a potential conflict of interest.

Publisher's Note: All claims expressed in this article are solely those of the authors and do not necessarily represent those of their affiliated organizations, or those of the publisher, the editors and the reviewers. Any product that may be evaluated in this article, or claim that may be made by its manufacturer, is not guaranteed or endorsed by the publisher.

Copyright (๑) 2022 Lin, Zong, Li, Tan, Sun, Zhang, Gan and Zeng. This is an openaccess article distributed under the terms of the Creative Commons Attribution License (CC BY). The use, distribution or reproduction in other forums is permitted, provided the original author(s) and the copyright owner(s) are credited and that the original publication in this journal is cited, in accordance with accepted academic practice. No use, distribution or reproduction is permitted which does not comply with these terms. 